\title{
KARL MARX' BELGISCHER FREUNDESKREIS (1 845-48): EINIGE NOTIZEN AUS BELGISCHEN ARCHIVEN
}

Es muss einem Belgier auffallen, dass im algemeinen die Sozialgeschichte seines Landes im Ausland wenig bekannt ist. Oft ergibt sich, dass man Belgier, die eine nicht unbeträchtliche Rolle gespielt haben, eigentlich nur dem Namen nach kennt.

Zum jüngsten Beispiel möchte ich die in den Jahren 1956 bis 1960 beim Dietz Verlag, Berlin, erschienenen Bände der neuen deutschen Ausgabe der Werke von Karl Marx und Friedrich Engels anführen, die auf der russischen Ausgabe fusst. Es handelt sich dabei nicht um eine nach streng wissenschaftlichen Gesichtspunkten angelegte Gesamtausgabe; die Arbeitsweise der Herausgeber wird jedoch in jedem Band vom Institut für Marxismus-Leninismus beim Z.K. der S.E.D. sorgfältig motiviert. Fussnoten und Anmerkungen der Redaktion zur Erklärung geschichtlicher Anspielungen, Personenverzeichnis usw. sind meist kurz und klar und nur leicht politisch gefärbt. Beim Personenverzejchnis aber fällt die blasse neutrale Charakterisierung der meisten Belgier auf. Auch kommen oft Irrtümer vor, weil man über die belgischen Verhältnisse ziemlich im Dunkeln tappt.

Wie kommt es, dass man im Ausland über den belgischen Anteil am gemeinschaftlichen Ausbau der sozialistischen Arbeiterbewegung im allgemeinen nicht gut unterrichtet ist? Jener Anteil war nicht gering, wenn man sich die Bevölkerungszahlen der in Betracht kommenden Länder vor Augen hält, und er erscheint noch grösser, wenn man dabei bedenkt, dass La Conspiration des Egaux, dite de Babeuf (1828) und das Manifest der kommunistischen Partei (1848) beide zu Brüssel entstanden (das erste wurde dort auch gedruckt); dass Brüssel mit seinen zahlreichen deutschen Flüchtlingen in den vierziger Jahren ein wirklicher Knotenpunkt der kommunistischen Bewegung, und mit den noch viel zahlreicheren französischen Verbannten vor und nach I 870 eine Druck- und Verlagszentrale illegaler Schriften war; dass Bakunin und Proudhon (um nur diese zwei grossen Namen aus einer ganzen Reihe meist französischer Flüchtlinge zu nennen!) mehrere Jahre in Belgien gewohnt haben, wo sie Freunde 
und Anhänger zurückliessen. Ich denke auch an Cesar de Paepes Beitrag zu der Ersten Internationale und zum Aufbau der Theorie des Kollektivismus; an die grossartige Ausbreitung des Genter Genossenschaftswesens; an die Zweite Internationale, die in Brüssel ihren Sitz hatte und an dessen Präsidenten Emile Vandervelde und Sekretär Camille Huysmans. Die zwischen den zwei Weltkriegen eine Sensation hervorrufenden theoretischen Schriften $A u$ delà du Marxisme, Die sozialistische Idee usw. eines Heinrich de Man dürfen wir noch hinzufügen, um ohne lächerlichen Nationalstolz behaupten zu können, dass Belgien eine Rolle gespielt hat, die anerkannt zu werden verdient.

Die Rolle der Association démocratique (1847-48) ist im allgemeinen besser bekannt, weil Marx bei der Gründung am i s. November 1847 zum Vize-Präsidenten gewählt wurde. ${ }^{1}$

Der Schatzmeister dieser Association démocratique, „ein Freund des Generals Mellinet" (F. Engels) heisst Felix Balliu, und nicht wie es oft falsch geschrieben wird, Ballin. Er war Gemäldehändler von Beruf; er wurde 1802 in Gent geboren und im August I 848 nach einer gescheiterten Invasion zum Tode verurteilt. ${ }^{2}$

J. L. Labiaux, ein Handelsreisender und ebenfalls ein Freund des französischen Generals Mellinet, des Ehrenpräsidenten der Association démocratique, war in den vierziger Jahren in den Arbeiterorganisationen (Volksmaatschappijen, Sociétés populaires) tätig: Agneessens in Brüssel, Artevelde in Gent, Breydel en De Coninck in Brügge und Zannekin in der kleinen Textilstadt Ronse (Renaix). Im April I 846 erliess Labiaux zusammen mit Pellering und anderen Genossen einen Aufruf zu einem Hungermarsch nach Brüssel: Oproep tot de ware Belgen, Appel au peuple belge, mit einem pathetischen Wort an die Soldaten am Schluss dieses Aufrufs. Der Auflauf wurde von den erschrockenen Justizbehörden unsanft bezwungen. Labiaux war es auch, der in der Association démocratique den Vorschlag machte, Marx' Rede über die Frage des Freihandels, gehalten am 9. Januar 1848, auf Kosten der Gesellschaft zu veröffentlichen.

1 L. Jottrand, Charles-Louis Spilthoorn. Evénements de $184^{8}$ en Belgique, Bruxelles I 872 .

L. Bertrand, Histoire de la Démocratie et du Socialisme, Bruxelles-Paris 1906 (veraltet, aber unentbehrlich).

Luc Somerhausen, L'humanisme agissant de Karl Marx, Paris 1946.

J. Kuypers, Bergop², Antwerpen 1962. - Ders., Geschiedenis van de socialistische Arbeidersbeweging, Antwerpen seit I 960 .

2 Unsere reichste Auskunftsquelle bilden die zahlreichen, hier meist zum erstenmal benutzten Akten der Justizbehörden aus den Jahren I 848-49 im belgischen Landesarchiv, T.C. Br. 667 . 
Arnold Battaille, Charles De Guasco, und die Brüsseler Schuhmacher Thomas Dassy (geb. 1819) und Jan Pellering gehörten dem selben republikanischen Agneessens-kreise an, der in den vierziger Jahren in Brüssel Hunderte von Meetings in den Arbeitercafés veranstaltete, soziale Manifeste und politische Broschüren in beiden Landessprachen herausgab, usw. Mit den deutschen Arbeitern unterhielten sie freundschaftliche Beziehungen und nach der Februarrevolution wurden sie gerichtlich verfolgt.

Charles Theodore De Guasco wurde im Jahre 1812 in Valkenburg in dem bei Holland verbliebenen Teil der Provinz Limburg geboren. Er war ein guter belgischer Patriot, Adjutant beim „Régiment des Guides"; später wurde er Beamter beim Athenäumpräfekt von Brüssel. Er kannte die beiden Landessprachen.

Der früh verstorbene (1858) Arnold Battaille war ein LithographFarbenmischer. Er wurde Herausgeber der demokratischen Zeitung Le Patriote belge (Redakteur Adolph Bartels) und zweiter Sekretär der Association démocratique. Er half Nicolas Coulon und J. Pellering bei der Redaktion ihrer Arbeiterzeitung Le Prolétaire (1855-68). Mit seinen Freunden, Anhängern von Proudhon oder Blanqui gründete er L'Affranchissement (1854): eine rationalistische, überwiegend proletarische Gesellschaft, die den Mitgliedern ein Zivilbegräbnis sicherte und unter den Arbeitern atheistische Propaganda trieb.

Die zwei Hauptfiguren aus dieser Periode des belgischen Frühsozialismus sind Jacob Kats (1804-86) und Jan Pellering ( 18 1 7-77).

Jener war in seiner Jugend Volksschullehrer und Weber. Im Alter von dreissig Jahren wurde er Mitglied einer Phalanx der Charbonnerie démocratique universelle, einer geheimen Organisation des berühmten Anhängers Babeufs, Camille Buonarroti. Einen absolut kommunistisch-utopischen Heilsstaat schildert Het aards Paradïs (Das Paradies [auf Erden], 2 Akte, 1 836). Seine Darsteller waren, ausser ihm selbst, andere Arbeiter und ihre Verwandten. Die Arbeiterzeitung Den waren Volksvriend ( $1836-40$ ) wurde das Organ der ebenso von ihm geg ründeten Flämischen Meetings (Brüssel 1836). Gegen diese politischen Arbeitervereine trat die Brüsseler Polizei sehr roh auf. Nach einer ersten Verurteilung empfing Kats eine bemerkenswerte Adresse der Londoner Chartisten an die belgischen, holländischen und rheinischen Arbeiter. In den vierziger Jahren mietete er ein Kaffeehaus mit Versammlungssaal und Bühne. Er wurde Fourierist, dichtete, sang und publizierte noch viel; aber nach den Ereignissen der Jahre I 848-49 zog er sich aus dem politischen Leben zurück und wurde Direktor des ersten flämischen Theaters in Brüssel (Tooneel der Volksbescbaving). Karl Grün, der ihn in seinem Arbeiterlokal besuchte, widmete ihm 
ein Kapitel seiner Sozialen Bewegung in Frankreich und Belgien (Darmstadt I 845). Die Deutsche Brüsseler Zeitung nannte ihn „den gewandtesten Volksredner Belgiens"; und Rosa Luxemburg bezeichnete ihn als „den originellsten vielleicht der internationalen sozialistischen Pioniere, den Schöpfer der ersten Arbeitervereine, der ersten demo kratischen Volkslieder, des ersten Volkstheaters in Flandren."

Jan Pellering (1817-77) war ein Schüler des Kats und ebenso ein unermüdlicher flämischer Volksredner. Beide haben mehrmals mit Marx von denselben Tribünen gesprochen und waren Mitglieder der Association démocratique.

August $\mathrm{I} 845$ erschien eine Broschüre $W$ at men is en wat men worden kan, eigentlich eine Rede von ,eenen Werkman Jan Pellering”. Dem Titel, aber nicht dem Inhalt nach, erinnert sie an W. Weitlings damals sehr bekanntes Büchlein Die Menscbbeit wie sie ist und wie sie sein soll. In den Jahren I846-48 wurde Pellering nicht weniger als viermal verhaftet.

Nachdem er später mit seinen Freunden die Begräbnisgesellschaft L'Afranchissement (1854) und die Zeitung Le Prolétaire (1855) gegründet hatte, trat er - selbstverständlich ohne Erfolg! - als erster Arbeiter-Kandidat bei den belgischen Parlamentswahlen auf. Als Schuhmacher widmete er sich der Handarbeit, aber er fand Gelegenheit, fortwährend seine theoretischen Kenntnisse zu vervollkommnen. In Anlehnung an Proudhon und Bakunin bekannte er sich zum Anarchismus. Vor und nach dem Haager Kongress der Ersten Internationale bekämpfte er das marxistische Organisationsstatut und jede zentralistische Struktur der nationalen oder internationalen Arbeiterbewegung. ${ }^{1}$

Von den Brüdern Adolphe (1802-62) und Jules Bartels (18 $15-55$ ) ist der erste am besten bekannt. März I 848 hat der „liberale” Jules im Gemeinderat der Stadt Brüssel über die polizeilichen Ausschreitungen gegen Marx und seine Frau interpelliert und eine amtliche Untersuchung errungen, die zur Entlassung des städtischen Polizeikommissars führte. Adolphe war ein zum Katholizismus bekehrter Protestant. Er war Mitarbeiter des Abbé de Lamennais in Paris in der Redaktion der Zeitung L'Avenir. Im Jahre 1830 behauptete er sich als energischer Patriot gegen die Holländer; er wurde der Geschichtsscheiber des Aufstands in den flandrischen Provinzen. Er verweigerte jede Belohnung für seine Verdienste und führte ein mühsames Journalistenleben. Er war ein bürgerlicher, misstrauischer Demokrat, der nur langsam eine persönliche Auffassung des Sozialismus entwickelte (Essai sur l'organisation du travail, 1842). Bartels und Kats wurden

1 Demnächst wird erscheinen: J. Kuypers, J.P., een vergeten redenaar en anarchist. (Verlag Ontwikkeling, Antwerpen). 
zugleich freigesprochen in einem politischen Prozess, der gegen sie angestrengt wurde, als sie sich dem Vorhaben der Regierung Leopolds widersetzten, einen Vertrag zu bestätigen, der den Verzicht auf Luxemburg und Limburg bedeutete. Anfänglich war Bartels Redakteur des Débat Social, organe de la démocratie; er verliess jedoch das Wochenblatt als es sich zum Fourierismus bekannte (1 846).

Von Marx' scharf polemischen Artikeln vom 19. Dezember 1847 und vom I3. Februar 1848 (beide erschienen in der Deutschen Brüsseler Zeitung) ist der erste ausdrücklich gegen den intrigierenden Bartels, der zweite gegen Lucien Jottrand gerichtet, der damals Redakteur des Débat Social und Präsident der Association démocratique war. ${ }^{1}$ Nach seiner Verhaftung und Ausweisung lobte Engels - und mit ihm wohl auch Marx - öffentlich einige belgischen Demokraten, namentlich auch Herrn Jottrand, die sich sehr anständig gegen die verfolgten Deutschen verhalten hätten. Ein spärlicher Briefwechsel Marx-Jottrand setzte sich noch jahrelang fort. ${ }^{2}$

Marx und Engels haben in Belgien mindestens zwei ausgezeichnete Mitarbeiter gefunden. Der erste, Philippe-Charles Gigot, war am 24. Dezember 18 I9 in Brüssel geboren und gehörte zugleich zum Freundeskreis Bakunins ${ }^{3}$ und Marx'. Er wurde Mitglied des Kommunistischen Korrespondenz-Komitees. „Die hiesige Adresse, an die Sie schreiben müssten", heisst es am 5. Mai 1846 im französischen Briefe von Marx an „mon cher Proudhon”, „,ist: M. Philippe Gigot, 8, rue Bodenbroek. Gigot wird auch die Briefe aus Brüssel unterzeichnen." Gigot kommt nicht nur auf der Liste des Deutschen Arbeitervereins vor; er war auch Mitglied des Vorstandes der Brüsseler Gemeinde (1 846-48) und vom 26. Februar - 3. März I 848 der Zentralbehörde des Bundes der Kommunisten. Noch im selben Jahre wird er, der Attaché au bureau de Paléograpbie im Innenministerium war, aus dem Staatsdienst entlassen. ${ }^{4}$ Gigot war ein tüchtiger Beamter, candidat en philosophie et lettres der damals jungen Brüsseler Universität, der nicht nur die beiden Landessprachen kannte, sondern auch deutsch und spanisch. Schon am 2 r. November 1849 bat der Reichsarchivar Gachard den Innenminister, ihn wieder aufnehmen zu dürfen. Wiewohl keine rechtmässige Ernennung möglich war - „des motifs particuliers m' empêchent pour le moment ... à cause des tripotages auxquels il a été mêlé" -, gelang es dem Reichsarchivar, ihn am I. März I 850

1 L. Somerhausen, op. cit., pp. 2 1 7-22 I.

2 La Pensée (revue) Nov.-Dez. I 958, Paris. - Uber L. Jottrand habe ich in der Biographie Nationale (Académie royale de Belgique XXX, 2) einen ausführlichen Aufsatz veröffentlicht.

${ }^{3}$ Confession, Paris 1932, pp. 78 u. 98 ; M. Nettlau, I, pp. 78-79 und Anm. 393.

4 Landesarchiv, Dossier administratif A. 1749-6; John Bartier, Le Mouvement démocratique à l' U.L.B. Socialisme (revue) Januar I 960 , Brüssel. 
provisorisch mit einer schwierigen Arbeit zu betreuen, und zwar mit dem Ordnen der'diplomatisch-finanziellen Korrespondenz des Königs Philipp II. bezüglich der Verwaltung der Niederlande. Ein königlicher Erlass vom 29. Oktober 1857 regelte endlich Gigots amtliche Stellung; sein Jahresgehalt betrug anfänglich $\mathrm{i} 800$ Franken und wurde am 21. März I 859 auf 2000 Franken gebracht.

Doch der fast dreissigjährige employé de première classe aux Archives du Royaume war seit langem krank. Der aus der Korrespondenz MarxEngels bekannte, in Belgien seit 1832 wohnhafte Deutsche Dr. Breyer (1 8 1 2-76) war sein Arzt. Am 4. September 1860 starb Gigot an einer Brustkrankheit in Bad Ems. Seine Hotelrechnung von 201 . I F Franken war nicht bezahlt. Ob das belgische Finanzministerium Gigots letzten Monatsgehalt von 165 Franken zur Begleichung verwendet hat, ist nicht sicher.

Philippe Gigot, der eine Wiener Volksschule besucht hatte, übersetzte verschiedene Erzählungen des berühmten flämischen Schriftstellers Hendrik Conscience ins Deutsche: Baes Gansendonck (1 8 50), Die hölzerne Clara (185I), Die blinde Rosa (185I), Der arme Edelmann (I851), Der Bauernkrieg (1853), Der Rekrut (1854) (erschienen bei Kiessling, Brüssel).

Victor André Tedesco war am 26. Juni 182 I in Luxemburg geboren. Also war seine Muttersprache Deutsch. Er war ein guter belgischer Patriot, der schon als Student leidenschaftlich gegen die Aufsplitterung seiner kleinen Heimat in eine belgische Provinz und ein holländisches Grossherzogtum auftrat (1838). Er studierte an der wallonischen Universität Lüttich (Dr. Jur. 1844); dort bestand er auch seine Probezeit in einer Advokatur. ${ }^{1}$ In der Brüsseler Association démocratique wie in der Lütticher Société Républicaine und für die Arbeiterzeitungen L'Owrier, L'Eclair (Lüttich), L'Atelier démocratique (Redakteure waren der deutsche Kommunist Ludwig Heilberg und der französische Blanquist Jacques Imbert, Brüssel) war Tedesco tätig. Er war ein gefeierter Redner. Wie Marx war er der Sobn eines Juristen. Seit I 847 war Tedesco Mitglied des Bundes der Kommunisten.

Als zweiter belgischer Delegierter nahm Tedesco im November I 847 an der Londoner Reise Marx' und Engels', an dem internationalen

1 A. Gaspar, Le Manifeste du Parti Communiste et le Catéchisme des Prolétaires de Victor 'Tedesco, in: Socialisme, septembre 1960, Brüssel.

Dieser Artikel war schon geschrieben (März I 962) als wir vom Institut für Marxismus und Leninismus, Moskau, dankbar eine ausführliche, kritische Studie in russischer Sprache von E. Kandel über die Verbreitung des ersten Programms des wissenschaftlichen Kommunismus empfingen (A kademisches und Auskunftsbulletin, 1962, Nr. 8, Moskau); darin werden die Bedeutung Tedescos und seines „Katechismus” eingehend erörtert. 
Meeting der Fraternal Democrats und an dem zweiten Kongress des Bundes der Kommunisten teil. ${ }^{1}$

Beim grossen Antwerpener Prozess im August 1848 behauptete er, ein beschlagnahmter Text eines Manifestes der kommunistischen Partei sei nur eine von ihm unternommene französische Übersetzung des deutschen Originals. Freunde hätten ihn darum gebeten, seine Arbeit sei jedoch nicht ganz fertig geworden.

Im Jahre 1848 wurde er dreimal verhaftet. Seine erste Verhaftung, nach einer leidenschaftlich demokratisch-republikanischen Rede in der Association démocratique, dauerte vom 28. Februar bis zum i 2. März, seine zweite exfolgte am 26. März. Zwischen beiden Verhaftungen war er in Brüssel. Zwei Tage nachdem er dort - den I9. März - aus Lüttich angekommen war, musste sein Freund Engels, der sich sehnte ihn wieder zu sehen, schon nach Paris abreisen. Die ersten Exemplare des Manifestes der Kommunistischen Partei sind am 20. März in der französischen Hauptstadt angekommen, folglich etwa an demselben Tag auch in Brüssel. Es ist also wahrscheinlich, dass Tedesco, der mit dem Gedankenkreis des Manifestes gründlich vertraut war, Ende März mit der - verloren gegangenen - ersten französischen Übersetzung begonnen hat. Und Engels war mit ihm einverstanden. Der arme Tedesco konnte jedoch seine Arbeit nicht zu Ende bringen, weil er schon am 26. März, wenn auch für eine kurze Zeit, und sofort nach der Risquons-Tout Affaire (29.3. I 848) zum dritten Mal verhaftet wurde.

Ein anderes, interessantes Problem stellt seine Broschüre Catécbisme du Prolétaire dar. Ende Dezember oder Anfang Januar 1849, ohne Verfasserangabe in den Almanach républicain pour l'année i $849 \mathrm{der}$ Redaktion einer Lütticher Zeitung, Le Peuple, aufgenommen, ${ }^{2}$ erschien der Text in demselben Jahre in einer Sonderausgabe mit zwei Umschlägen ( $18^{\circ}, 16$ p.) bei der gleichen Druckerei A. Charron. Aber diesmal führte der erste Umschlag den Namen des Verfassers: par $V$. Tedesco, Détenu politique. Donnerstag den ro. Mai 1849 kündigte die Zeitung eine neue Auflage von zehntausend Exemplaren an. (Preis 6 centimes.) Der Dichter Ferdinand Freiligrath, der mit seiner jungen Gattin ein halbes Jahr (1844-45) in Ostend und Brüssel gelebt hatte und der Redaktion der Neuen Rheinischen Zeitung angehörte, soll die

1 Brief von Engels an Marx vom 23./24. November 1847 und Deutsche Brüsseler Zeitung, 9. Dezember 1847 .

${ }^{2}$ Le Peuple erschien zu Lüttich vom 4. Februar bis zum 30. Dezember I849. Das Musée de la vie wallonne, Lüttich, besitzt einen Teil dieses einzigen Jahrganges (Nr. 236 ). In derselben 10. Mainummer 1849 trifft man eine zweite merkwürdige Anzeige an: "Sous. presse: Garanties de l'Harmonie et de la Liberté par Guillaume Weitling. Aus dem Deutschen übersetzt von den Redakteuren der Zeitung Le Pcuple. Preis: 95 centimes." Im Monat Oktober (Nr. 84 und 85) hat Le Peuple zwei Feuilletons aus dieser Übersetzung gebracht: De l'Etat militaire, über das Soldatenleben in Preussen. 
Schrift ins Deutsche übersetzt haben: „Katechismus des Proletariers Von Tedesco". Sie wurde ohne Angabe des Namens des Übersetzers im Verlag des sozialistischen Literatur-Comptoirs, i 849, London und New York veröffentlicht. (Preis I Penny - 2 Cents - I Rgr.) Diese gute, wortgetreue Übersetzung, in der sogar die Numerierung der Fragen verbessert wurde, beweist jedenfalls, dass jener Kölnische Freundeskreis den belgischen Ableger des Manifestes schätzte. In einer Fussnote erwähnt E. Kandel, diese Übersetzung von F. Freiligrath sei mit Hilfe von Karl Schapper und Joseph Weidemeyer zustande gekommen und insgeheim, nicht in London, sondern in Frankfurt 1850 gedruckt worden. ${ }^{1}$

Meiner Meinung nach hat der am 30. August 1848 zum Tode verurteilte Tedesco schon am Anfang seiner schweren Kerkerhaft in Antwerpen, wo er bis zum Ende des Monats Januar i 849 blieb, seinen Katechismus geschrieben; selbstverständlich ohne irgendwelches Hilfsmittel, weil die Justizbehörde seine Dokumente beschlagnahmt hatte. Er atmete in einer hauptsächlich von Marx' überragender Persönlichkeit beeinflussten Gedankenwelt. Vor und nach der Londoner Reise, wie während der Versammlungen selbst, hatte er persönliche Bemerkungen gemacht und Thesen verteidigt. Er erinnerte sich wörtlich der Dokumente der Zentralbehörde des Bundes der Kommunisten. Insbesondere schwebte ihm jener Entwurf in Katechismusform vor, den Friedrich Engels seinerseits in Paris für das Manifest verfasst hatte: die Grundsätze des Kommunismus, ${ }^{2}$ die er auf der Londoner Reise gelesen und mit seinen beiden verehrten Freunden ausführlich besprochen hatte.

Nicht nur nach der Form, mit Fragen und Antworten, stimmt die Broschüre von Tedesco mit Engels' bekanntem Entwurf überein. Ihrem Inhalt nach gehören drei Viertel zum Gedankenkreis des Entwurfs. Fast wörtlich enthält sie mehrere der gleichen Thesen.

In seinem schon genannten Brief vom 23./24. November I 847 hatte Engels den Gedanken an einen Katechismus aufgegeben. Wahrscheinlich hatte Marx ebenfalls einen Entwurf vorbereitet. Was haben die drei Freunde während ihres tagelangen Beisammenseins auf ihrer Hin- und Rückreise und in der englischen Hauptstadt untereinander wie mit den Kollegen des Kommunistenbundes sonst diskutiert und

\footnotetext{
1 Mit Kandels Behauptung, der Name des Verfassers komme zum ersten Mal in dieser deutschen Übersetzung (1850) vor, sind wir nicht einverstanden. Die erste, namenlose Ausgabe, als letzter Teil des Lütticher Almanachs, datiert zweifellos vom Ende Dezember I848, oder spätestens vom Anfang Januar I849. Die zweite Ausgabe ist im Jahre 1849 sichtbar neu gesetzt worden in derselben Druckerei Charron und führt den Namen V. Tedesco, Détenu politique.

2 Karl Marx/Friedrich Engels, Werke, Bd. 4.
} 
verabredet? Welchen Anteil an der Diskussion und an der Vorarbeit hat der fast sechsundzwanzigjährige Jurist Tedesco genommen? Die von Engels aufgegebene Katechismusform (Grundsätze des Kommunismus) hat er sicher verteidigt, weil sie ihm für das belgische Proletariat tauglich $\mathrm{zu}$ sein schien. ${ }^{1}$ Und nach dem Erscheinen des Manifestes seiner beiden bewunderten Freunde hat der einsame, opferbereite, von Engels hochgeschätzte ${ }^{2}$ Tedesco eben in dieser populären Form einen Katechismus verfasst.

„Qu' est-ce qu'un prolétaire?” heisst es in Tedescos Broschüre. „Le prolétaire est l' esclave du capital; - il n'a point de pain s' il n' a point de maître."

Wodurch unterscheidet sich der Proletarier vom Sklaven? - Und vom Neger? fragt Engels (wie die Vorbereitungsdokumente des Kommunistenbundes, und später das Manifest selbst). Tedesco stellt dieselben Fragen und seine Antworten sind ähnlich:

„Le prolétaire et le nègre, pour vivre, dépendent l'un et l'autre d'un maître.

Le prolétaire, à la différence du nègre, peut quitter librement le maître qui l'emploie, sauf à mendier ou à voler s'il n'en trouve pas un autre qui puisse ou veuille l'occuper.

Le prolétaire et le nègre, en changeant de maître ne changent point de sort, l'un reste toujours l'esclave, l'autre toujours prolétaire, quelles que puissent être la bienveillance et la philanthropie de ceux qu'ils servent.

Le nègre est esclave de l' homme.

Le prolétaire est esclave du capital."

Der Proletarier, schreibt Engels, arbeitet mit Produktionsınstru-

1 In seinem bemerkenswerten Aufsatz (op. cit.) untersucht E. Kandel, welche Ideen Tedesco von seinem genialen Freund Marx entlehnt hat (in der „Misère de la Philosophie” und den Vorträgen im Brüsseler Deutschen Arbeiterverein, aus denen „Lohnarbeit und Kapital”, sowie der Entwurf zu "Arbeitslohn” entstanden). Ohne Zweifel hatte Tedesco Marx' Arbeiten gelesen; aber er war bei den beiden Vorträgen (Dezember 1 847), deren Text erst später in der Neuen Rheinischen Zeitung abgedruckt wurde, wohl nicht anwesend. Er wohnte damals in Lüttich, roo $\mathrm{Km}$ von Brüssel entfernt, und gehörte nicht dem Arbeiterverein an: nur Gigot kommt als einziger Belgier auf Wolfs Liste der Mitglieder vor. Kandels Jagd nach direkten Einflüssen von Marx auf 'Tedesco ist interessant und reichlich lohnend. Doch dürfte in einigen Punkten vielleicht eher von einer gemeinschaftlichen Gedankenwelt im Bund det Kommunisten als von einer direkten Übernahme Marxscher Ideen die Rede sein. Das Manifest selbst ist, dem Inhalt nach, einigermassen Gemeingut. - Weiter behauptet E. Kandel, Tedesco habe schon vor der Londoner Reise (Nov.-Dez. I 847) nicht nur die meisten seiner Thesen niedergeschrieben, sondern sogar seinen Katechismus komponiert. Dies ist eine Arbeitshypothese, ohne irgendwelchen festen Beweis.

2 Briefe vom 15 . und 24. November 1847. 
menten eines andern für Rechnung dieses andern, gegen Empfang eines Teils des Ertrages.

„Pour le prolétaire, point de pain sans travail, point de travail sans instruments de travail. Or, dans l'organisation actuelle de la Société, les instruments de travail représentent, par eux-mêmes, une valeur tout aussi inaccessible à l'ouvrier que le capital nécessaire pour les exploiter."

Und weiter (Frage 3 I) lesen wir bei Tedesco: „L' Etat seul peut et doit devenir le banquier des prolétaires. Pour sauver la Société de l' abîme que lui creuse la concurrence, ce n' est pas trop de toutes les ressources, du concours de toutes les forces de l' Etat."

Eine der wichtigsten Massregeln ist für Engels: Zentralisierung des Kreditwesens und des Geldhandels in den Händen des Staats durch eine Nationalbank mit Staatskapital bei gleichzeitiger Aufhebung aller Privatbanken. Im Manifest wurde dies als ein Mittel zur Umwälzung der ganzen Produktionsweise noch kürzer ausgedruckt: „:Zentralisation des Kredits in den Händen des Staats durch eine Nationalbank mit Staatskapital und ausschliesslichem Monopol."

In Antwort auf seine vierte Frage sagt Tedesco: „Entre le maître qui achète et le prolétaire qui vend le travail, il se fait une transaction forcée qui est le salaire, lequel représente, par conséquent les conditions de la vie du prolétaire."

\section{Die s. Frage von Engels lautet:}

„Unter welchen Bedingungen findet dieser Verkauf der Arbeit der Proletarier an die Bourgeois statt? - Antwort: Die Arbeit ist eine Ware wie jede andere ... Der Preis einer Ware ... ist aber im Durchschnitt immer gleich den Produktionskosten dieser Ware. Der Preis der Arbeit ist also ebenfalls gleich den Produktionskosten der Arbeit. Die Produktionskosten der Arbeit bestehen aber in gerade so viel Lebensmitteln, als nötig sind, um den Arbeiter in Stand zu setzen arbeitsfähig zu bleiben und die Arbeiterklasse nicht aussterben zu lassen."

„Le travail, en tant qu'il se vend et s'achète, est une marchandise dont le prix s'appelle salaire. ... Le salaire ou le prix du travailmarchandise, dépend des frais qu'il faut faire pour produire le travail, c'est-à-dire des frais que nécessite l'entretien d'une race d'ouvriers.

De même que le prix d'une marchandise baisse lorsqu'il en coûte moins pour la produire; de même baisse le prix du travail-marchandise lorsqu'il en coûte moins pour entretenir l'ouvrier.

Que l'on découvre un aliment moins coûteux que le pain et les pommes de terre, et aussitôt le salaire baissera en proportion."

Der auffallende Parallelismus zwischen beiden Autoren könnte 
leicht weiter durchgeführt werden; die Ideen eines Kampfes von Klasse gegen Klasse, der anarchistischen Konkurrenz, von Proletatiern, die nichts zu verlieren haben:

„Alors n'ayant rien à perdre et rien à regretter, comment pourrions-nous ne pas jeter notre vie dans la balance sociale, pour en rétablir l'équilibre?

Vous regardez les prolétaires comme constituant une véritable classe dans la société? - Oui. Deux classes sont en présence l'une de l'autre: les riches et les prolétaires. Entre elles, il y a antagonisme..."

Engels setzt auseinander (und wieder folgt ihm darin das Manifest drei Monate später), wie die Bourgeoisie die Macht der Aristokıatie, des Adels und das absolute Königtum vernichtete, wie sie die konstitutionelle Monarchie einführte sowie ein Repräsentativsystem, nach dem nur diejenigen, welche ein gewisses Kapital besitzen, Wähler sind.

Tedesco sagt: „Après la victoire, la bourgeoisie, forte de ses richesses et de ses lumières,... établit la souveraineté des bons propriétaires, des industriels, des commerçants, de la propriété en un mot...

Dans cette rénovation de la société quel fut le sort de la royauté? Renversée par le peuple, la bourgeoisie la restaura sous le nom de royauté constitutionnelle, etc. etc."

Jedoch bleibt der Unterschied zwischen Engels' Entwurf und Tedescos Katechismus gross. Beide fussen auf gemeinsamen Einsichten, Gesprächen, Diskussionen; aber der erste ist ökonomischwissenschaftlich stärker motiviert und mehr auf die Gesamtheit der Probleme gerichtet. Der lebendig begrabene Tedesco hat seinen Katechismus selbständig ausgearbeitet und als Schriftsteller einen besonders leidenschaftlichen Ton getroffen, der in diesem Ausmass dem soliden Entwurf ebenso fremd ist wie dem Kommunistischen Manifest selber. So wiederholt er z.B. mit grossem stilistischem Erfolg fünfzehnmal den Satz: „Les proléraires ne le feraient pas”, oder "Les prolétaires ne le permettraient pas”. In einem äusserst pathetischen Anruf wiederholt er dreimal: „A quoi penses-tu, ô prolétaire?" Er ist mehr Redner und Jurist als Volkswirtschaftler und zeigt sich öfters als ein richtiger Achtundvierziger. Dies geht hervor aus seiner Verherrlichung der revolutionären Dreieinheit Liberté, Egalité, Fraternité als Grundlage für die soziale und demokratische Republik wie aus seiner romantischen Schilderung der schmutzigen Arbeiterviertel unserer Grossstädte oder aus der pathetischen Eloquenz seiner Schlusszeilen. Dort erreicht er einen rührend-lyrischen Schwung, der der logischen Struktur des Büchleins und dem Gleichgewicht seiner Argumentation vielleicht schadet, der einem aber den Atem raubt, wenn man dabei bedenkt, dass dieser aus dem Antwer- 
pener Kerker Saint André geschmuggelte Katechismus wie ein Schrei aus dem unheimlich finsteren Gefängnisfort in Huy an der Maas erklang, wo Tedesco vom Ende Januar 1849 bis zum 6. Januar I 854 verbleiben sollte ${ }^{1}$ : eine von einem erschütterten, aus rein politischen Gründen zum Tode verurteilten Idealisten erhobene Anklage. „Politischer Gefangener" stand auf dem Umschlag der Lütticher Broschüre. Wir können uns den Eindruk vorstellen, den dieser Schrei aus dem Totenreich auf dem Maasfelsen auf die Arbeiter gemacht hat. Es quillt eine Ttäne, die Fäuste ballen sich...

„Artisans de notre infortune, laissez passer la justice des prolétaires.

\section{Liberté, Egalité, Fraternité.}

Drapeau de nos pères marchant à la destruction de la Société féodale, reçois le serment du prolétaire. Dans sa lutte ardente contre l' aristocratie nouvelle, protège son coeur de toute défaillance. Prophétie de l' avenir, éclaire son intelligence et préserve-la des promesses décevantes de ses ennemis.

Et toi, prolétaire, mon frère, enfant de toutes les douleurs, que ta propagande, incessante comme ton travail, réponde enfin à tes souffrances. Que cette force que tu épuises au service d'un maître vienne en aide à celle de tes frères les prolétaires.

De cette sainte alliance surgira, une et indivisible

\section{LA REPUBLIQUE DEMOCRATIQUE ET SOCIALE."}

$$
\text { ** }
$$

Berichtigungen zweier Irrtümer in der grossen Berliner Ausgabe der Marx/Engels Werke folgen hier zum Schluss. Es handelt sich um zwei amüsante Versehen lokaler Art.

In der Neuen Rheinischen Zeitung vom 3. September I848 publizierte Engels empört einen Artikel über die siebzehn Antwerpener Todesurteile „aus Veranlassung der lächerlichen Geschichte von Risquons-Tout", einer gescheiterten französischen Invasion. In diesem Scheinprozess ,stellte man die Angeklagten vor die Antwerpener Geschworenen, vor die Elite jener flämischen Faronaturen, ... vor jene Stockfischhändler, die im kleinbürgerlichsten Nützlichkeitskram, in der kurzsichtigsten, schreckhaftesten Profitmacherei lebenslänglich dahinvegetieren".

In einer Fussnote „erklärt” die Redaktion das Wort „Faronaturen”

1 Victor Tedesco ist am 28. Mai 1897 in Arlon gestorben, wo er eine Rechtsanwaltspraxis hatte und Führer der liberalen Partei geworden war. 
als Glückspielernaturen. Dürfen wir darüber ein wenig lächeln? Faro war in Belgien bis zum ersten Weltkrieg ein beliebtes und billiges Braunbier, eine gezuckerte Mischung von Lambik und Mars. Lambik trinkt man heute noch, meistens als edles Flaschenbier: Geuzelambik oder Kriekenlambik. Mars dagegen braut man nicht mehr. Wie die meisten lokalen Getränke ist es der Industrialisierung und Normalisierung erlegen. Faronaturen sind also gemütliche, kleinbürgerliche Biertrinker.

In einem anderen Artikel, „Der Musterstaat Belgien”, in der Neuen Rheinischen Zeitung vom 7. August I 848, untersuchte Marx die wirtschaftliche Stagnation in dem monarchischen Eldorado mit der vielgepriesenen liberalen Verfassung. ${ }^{1}$ Die Zeitung L'Observateur hatte das Programm der patriotischen Septembertagefeier „um ein neues Vergnügen vermehrt, um eine Prozession - einen Ommeganck General - zu Ehren des Doudou aus Mons, des Houplala aus Antwerpen und des Mannequin Pisse aus Brüssel."

„Bekannte Denkmalsfiguren dieser Städte”, lehrt uns die Fussnote der Berliner Redaktion. Das weltbekannte Manneken Pis ist wirklich ein ... Denkmal. Der Doudou aus Mons dagegen ist nur ein schrecklicher Drache, den man jedes Jahr in einer Prozession umherfährt. Den ganzen Weg entlang probieren die Zuschauer um die Wette einige Schweifhaare zu erobern, weil diese Glück bringen sollen; und seit Jahrhunderten gibt es auf der Grand' Place in Mons einen tumultuarischen Kampf zwischen dem Ungeheuer und einem immer siegenden Sankt Georg, bei dem ein uraltes Volkslied gesungen wird: „C'est l'Doudou, c'est l'Doudou ... de Saint Georges qui va".

Der Houplala aus Antwerpen weist auf eine gleichartige Puppe hin, die Opsignoorke heisst. Seit dem fünf- oder sechzehnten Jahrhundert heissen die stolzen Einwohner der Handelsstadt an der Schelde Sinjoren. Bei dem Karnevals-Ommeganck "spielt" man Opsignoorken: vier Männer tragen ein grosses Segeltuch, worauf sie an jeder Strassenecke eine symbolische Puppe Sprünge machen lassen. Die Leute singen und rufen dabei; „Ajop, ajop, Sinjoorken, houplala!”

Mit einem Denkmalsbegriff haben diese Puppen, der kriegführende Doudou ebensowenig wie der leutselige Opsignoorke-Houplala, gar nichts zu tun. Sie zeigen dagegen, dass Marx Belgien und Holland kannte, auch manches von ihren Volksbräuchen sowie von der flämisch-holländischen Landessprache.

\footnotetext{
1 Zwanzig Jahre später, in einer Fussnote zum dreiundzwanzigsten Kapitel des Kapitals, hat Marx aufs neue der aus England stammenden Lüge: „Belgien als das Paradies der Arbeiter" den Boden eingeschlagen. Er zitierte Statistiken eines belgischen Nationalökonomen E. Ducpétiaux und ein Manifest (1 859) der Brüsseler "Maatschappij Vlamingen, vooruit!" als charakteristisch für die Lage ,in diesem Paradies der Kapitalisten”.
} 